\title{
NANO-NETWORK CONDUCTING POLYMERS AS SUPERIOR ELECTRODES FOR SUPERCAPACITORS
}

\author{
MOHAMMED A. IBRAHEM \\ Dept. of Physics, College of Science, University of Duhok, Kurdistan-region-Iraq
}

(Received: July 3, 2017 ; Accepted for publication: August 28, 2017)

\begin{abstract}
The characteristics of supercapacitor with a series of 3,4-alkylenedioxythiophene (XDOT) derivatives through the controlling of the ring size and substitutions variation have been investigated. The results indicated that more-open morphology of the polymers produced the excitant of the bulky group on the monomer, which produce the ionic transfer during the redox reaction leading to higher specific capacitance and better stability. Poly(3,4-propylenedioxythiophene) PProDOT shows a highest specific capacitance $\left(125 \mathrm{~F} \mathrm{~g}^{-1}\right)$ which can maintain more than $85 \%$ of its original values after $1 \mathrm{k}$ cycles compared with other polymers.
\end{abstract}

KEYWORDS: Conducting polymer; supercapacitors; morphology; Stability; nanofibers.

\section{INTRODUCTION}

$\mathbf{R}^{2}$ ecently, a great interest has been given to supercapacitors which is known also as ultra-capacitors are another type of storage devices with high power density and stability and rapid charged/discharge. Therefore, it is becoming a very good candidate as storage device in many applications such as vehicles and electronic devices. Because of their higher storage capacities more than that of conventional capacitors, environmentally friendly, and safety that can operate in a various range of temperatures (Beidgahi and Gogotsi 2014). Depending on the mechanism of charge storage, either the supercapacitors will store the energy at the interface between the electrode and electrolyte and it is kwon as electrical double layer capacitors (EDLCs) or it will store the energy through the utilization of the faradic reactions and known as pseudocapacitors (Beidgahi and Gogotsi 2014 , Simon and Gogotsi 2008, Xu et al., 2013, and Le et al., 2013).

So far, different materials have been employed as electrodes in the fabrication of supercapacitors like, polymer composites (Su et. al, 2013 and Liu et. al, 2013), conducting polymers (Yang et. al, 2013 and Reddy et. al, 2014), carbon materials (Kim et. al, 2013 and Xiao et. 1, 2014), and some metal oxides (Saravankumar et. al 2012 and Zhu et. al, 2014). Among them, conducting polymers are the most optimistic materials, according of their extraordinary advantages in the combination of high energy storage, high ability of reversibly oxidization and reduction, easy to be prepared, mechanical flexibility and low cost (Snook and Best, 2011). Among the conducting polymers, 
has received a great attention in many applications. In particular, poly(3,4-ethylenedioxythiophene) (PEDOT) gets a great interest, specially, the emphasize has been given to the enhancement of their specific capacitance (Wu et. al, 2010). For example, a $140 \mathrm{~F} \mathrm{~g}^{-1}$ is achieved with PEDOT nanotube arrays (Liu et. al 2008). Also, by modulating the morphology under different surfactant concentrations, the specific capacitance enhanced from less than 40 to more than $100 \mathrm{~F}$ $\mathrm{g}^{-1}$ ( $\mathrm{Li}$ et al. 2010). A $106 \mathrm{~F}^{-1}$ specific capacitance achieved from PEDOT/carbon composite (Kelly et al. 2009). However, the serious degradation of polymers in the ambient condition, they perform relatively poor stability and limit their uses in many applications.

Therefore, an intensive study has been given to such issues proposing different strategies. For example, by using different electrolytes (ionic liquids), the degradation minimized and thereby its stability increased tremendously which is be attributed to its non-volatility, non-flammability, wide potential windows and high thermal/electrochemical stability (Torimoto et. al, 2010). Moreover, the issue of short cycle life is originated mainly from the intrinsic property of polymer films. The migration of counter ions of the conducting polymers is the fundamental characteristic of a redox capacitor. Based on this viewpoint, it is possible to enhance the reversibility of ionic transport by building the nanomorphology for the electroactive layer. Here in this work, we use XDOT to prepare a highly porous polymer films in nano-scale via electrochemical deposition method in order to study mechanism of the charge carrier transfer within the polymer. It is demonstrated that the stability of capacitor is obviously improved with porous structure polymer films.

\section{Experimental Section}

\section{Preparation of polymer electrodes}

Polymerization experiments were carried out in a three-electrode electrochemical cell with a platinum foil counter electrode and an $\mathrm{Ag} / \mathrm{Ag}^{+}$ reference electrode $\left(0.01 \mathrm{M} \mathrm{AgNO}_{3}\right.$ and $0.1 \mathrm{M}$ $\mathrm{TBAClO}_{4}$ in $\mathrm{ACN}$ ). Nickel mesh was used as working electrode. The monomer solution contained $0.01 \mathrm{M}$ EDOT or its derivatives in a 0.1 $\mathrm{M} \mathrm{LiClO}_{4} / \mathrm{ACN}$ solution. The deposition of PXDOTs were controlled by an Autolab potentiostat/galvanostat (Eco Chemie, model PGSTAT30). The polymer thin films were electropolymerized onto the Nickel mesh by applying a potential at $1.1 \mathrm{~V}$ with a charge capacity of $2 \mathrm{C} \mathrm{cm}^{-2}$.

\section{Characterization}

In order to probe out the morphology of the prepared polymers film a scanning electron microscopy (SEM, Hitachi S-4700) was used. The stoichiometry studies were performed using X-ray diffraction (XRD, Philips X'Pert). Electrochemical Quartz Crystal Microbalance (EQCM) investigations were carried using Auto-lab potentiostatic/galvanostatic (Seiko EG\&G, QCA917). Based on the Sauerbrey equation (Liu et. al, 2008), the change in the mass with the active area were calculated.

\section{RESULTS AND DISCUSSION}

The structures of PXDOT polymers are shown in Scheme 1. The morphology changes of the as synthesized polymers with increase in the ring size is shown in figure 1 . It is clear that the 
porosity of the polymer films increased with increasing the ring size (from two to three carbons or when the carbon side chains are grafted). This can be attributed to the difference in the ring size and its substitution, which effects on the physical properties of the as-deposited polymer significantly (Ren and Pickup, 1994). When the ring size is increased the coplanar stacking becomes weaker due to the larger twisty angle of the ring. Furthermore, the bulky substitutions are on opposite sides of the thiophene plane which serves to separate the polymer chains. Compared with the dense PEDOT film, the PProDOT and PProDOT-Et ${ }_{2}$ reveal a fiber-like and highly porous structure. The diameters of the nano-porous in the films are about 5-10 nm, 50-70, and 10-20 nm for PEDOT, PProDOT and PProDOT-Et 2 , respectively. It is believed that the porous morphology allows for faster ion movement, which is often the rate-limiting process, during the redox reaction.

The structure of PXDOT was also investigated by the XRD analysis. The PXDOT powder was collected from electrochemical deposition. In figure 2, the PEDOT features the diffraction peaks located at $6.4,12.8$ and $26.3^{\circ}$, which can be assigned to (100), (200) and (020) reflection, (Ling et. al, 2014) respectively, indicating the existence of a crystalline phase. The peak at $2 \theta$ at $6.4^{\circ}$ is considered to be in relation to the inter-chain distance within the stack which is around $1.38 \mathrm{~nm}$. Another diffraction centered at $26.3^{\circ}$ for PEDOT is corresponded to a spacing of $0.33 \mathrm{~nm}$. For the PProDOT, it can be seen a shift of the (020) peak position to a lower angle $\left(26.3^{\circ}\right)$ leading to a larger stacking distance $(0.35 \mathrm{~nm})$.
This is due to the disruption of the coplanar stacking by the larger ring size. In the case of PProDOT-Et 2 , the (100) peak disappear and the (020) peak shift further to $24.8^{\circ}$ implying the ethyl substitution disrupt the crystallinity, significantly. The features are in line with our SEM results. The poor stacking of the PProDOT-Et $_{2}$ results in a decrease of charge carrier hopping between the polymer chains leading to a higher internal resistance (Chen and $\mathrm{Wu}, 1995$ ).

Figure 3 shows the CV curves of the polymer films deposited on Ni-mesh in $1 \mathrm{~mol} \mathrm{LiClO}_{4}$ solution at $10 \mathrm{mV} \mathrm{s}^{-1}$ scan rate. To obtain the optimized electrical conductivity and electrochemical reversibility of PXDOT, the cyclic voltammetry scans were performed in a potential range zero to one Volt. As seen from figure 3, the $\mathrm{CV}$ curves of PEDOT and PProDOT have a rectangular shape, indicating a good capacitive behavior. In contrast, for the PProDOT-Et ${ }_{2}$, the CV shape is distorted in the scanned range between 0 and $1.0 \mathrm{~V}$, indicating higher series resistance. The series resistance combines three main contributions, the bulk electrical resistance of the polymer film, the bulk electrolyte resistance (In this case, this term is a constant because of the same electrolyte) and the electrolyte resistance in the pores of the polymer matrix (Lin et. al, 2009). As shown in the figure 2, because of the dimethyl substitution, inter-chain charge movement is proposed to be hindered, and the conductivity of the PProDOT-Et ${ }_{2}$ is lowered. In earlier study, (Huang et. al, 2008 ) they also found the thickness of PProDOT-Et ${ }_{2}$ is much larger compared with those of PEDOT and PProDOT 
under the same deposited charge density. The thicker polymer films are due to its loose structure which also leads to higher internal resistance. Furthermore, the extremely porous structure of the PProDOT-Et ${ }_{2}$ also results in ion sieving effect leading to an increase in ionic resistance in the pore of polymer film (Ren and Pickup, 1994).

The Galvanostatic cycling is performed at a constant current density $(1.5 \mathrm{~A} / \mathrm{g})$. Figure 4 shows the charge/discharge curves of both PEDOT and PProDOT polymers are linear with constant slopes of the curve, indicating excellent capacitive behavior. The specific capacitance is evaluated from the equation 1.

$C=I \Delta \mathrm{t} /(m \Delta V)$

where $I$ is the applied current and $m$ is the mass of each electrode. The discharge specific capacitances of the three polymer electrodes are 87, 126 and $46 \mathrm{~F} \mathrm{~g}^{-1}$ for PEDOT, PProDOT and PProDOT-Et ${ }_{2}$ respectively, that are comparable to the results from current-voltage at $10 \mathrm{mV} \mathrm{s}^{-1}$. A higher capacitance of PProDOT compared with the one of PEDOT is contributed to its moderately porous structure. It is known that the contact between the electrolyte and the active layer can be moderated by nanostructure. Furthermore, the nanofiber structure of PProDOT also will provide larger surface area than that what PEDOT will provide and thereby reduce the diffusion distances for ions. Compared to PProDOT, the specific capacitance of PProDOT-Et $t_{2}$ decreased dramatically. This can be explained by (i) the higher molar mass of ProDOT-Et $_{2}$ monomeric unit and (ii) its too porous morphology leading to larger resistance and ion sieving effect. These results could be further supported by investigating the electrochemical impedance spectroscopy (EIS) as shown in figure 5 . The impedance spectra of PXDOT films were recorded under zero applied voltage versus $\mathrm{Ag} / \mathrm{AgCl}$ within frequency range of $10^{4}$ to $10^{-2} \mathrm{~Hz}$. In a typical EIS, it is composed of two lines a semicircle and an inclined straight line in both high and low frequency regions respectively. Where, the semicircle represent the charge transfer at electrode/electrolyte interface, while the inclined straight line represents the diffusion limiting step in electrochemical processes. Here in figure. 5, it is hard to recognize a well-defined semicircle, which indicating that, the charge transfer resistance is very low and therefore indicating a very highly conductive polymer films that well adhesive with the electrode. In addition, further information could be gotten from the ionic transport at high frequency real axis intercept $\left(R_{\text {high }}\right)$ and the low frequency limiting real impedance $\left(R_{\text {low }}\right)$ as follow

$R_{\text {low }}-R_{\text {high }}=1 / 3 R$

Ionic resistance values are 2, 2.1 and 8.1 $\Omega$ for PEDOT, PProDOT and PProDOT-Et $t_{2}$, respectively. The higher ionic resistance of PProDOT-Et ${ }_{2}$ may be raised from the ion sieving effect that is observed with a small pore size. In general, the extra contribution from Faradaic reaction will effect directly on the value of the obtained capacitance from the CV measurements (M. Ates 2009). Furthermore, the specific capacitance, which is known as low frequency capacitance that could be calculating from the reciprocal slope of the negative of electrochemical impedance with $(2 \pi \mathrm{f})^{-1}$ curve (inset of figure 4) by using equation 3 below. 
$-\mathrm{Z}^{\prime \prime}=\frac{1}{\mathrm{C}_{\mathrm{E}}}(2 \pi f)^{-1}$

The calculated specific capacitance of PEDOT, PProDOT and PProDOT-Et ${ }_{2}$ are 72, $106,32 \mathrm{~F} \mathrm{~g}^{-1}$, respectively. Although the specific capacitance calculated from EIS is smaller than that from CV measurement, it still reveals the PProDOT has larger capacitance than the others.

It is important to investigate the ionic exchange process in polymer films. Figure 6 shows $\Delta \mathrm{m}$ vs. the current-density plots of PXDOTs scanned between zero and one Volt for the first 100 cycles. For the PXDOT films, at potentials higher than $0.6 \mathrm{~V}$, continuous increase in the mass can be observed. This increase can be correlated to the trap of anions, solvated species and the solvent (Hillman et. al, 2007). This indicates that both charge carrier types can easily transfer into the electrodes but hardly can eject during redox process (Plieth et. al, 2006). For PEDOT polymer, within the first 100 cycles, the accumulation of mass was $3350 \mathrm{ng}$, while a very small amount of PProDOT (430 ng) and PProDOT-Et $_{2}(290$ ng). This smaller trapped amount of cations and solvated species in both PProDOT and PProDOT-Et ${ }_{2}$ compared to PEDOT is a clear evident of more reversible through the ions exchange which is may be due to the more porous morphology.

The degradation in polymers is a very serious issue that should be taken in consideration for many applications specifically for supercapacitors. Fig. 6d shows the charge capacity variations of polymer electrodes compared with the $1^{\text {st }}$ cycle as a function of cycle number. Because of the high degradation of polymers due to the irreversibility of ionic transfer, it can be seen the PXDOTs electrodes shows decay in the charge capacity. Even though, PProDOT and PProDOT-Et ${ }_{2}$ shows more stability than PEDOT. The specific capacitance decade slowly from 125 to 45 for PProDOT, 105 to 36 for PProDOT-Et 2 , and 87 to $31 \mathrm{~F} \mathrm{~g}^{-1}$ for PEDOT. During the redox process, both cations and anions compensate in the PXDOTs electrodes. Also, for all polymer electrodes, a transpiration of few ion pairs was found (figure 6). When the ion pairs trapped into polymer, it is very hard to accommodate other ions in order to achieve electroneutralization that will effect directly on the polymer electroactivity (decreased gradually). Therefore, it can be found with larger increased mass the charged capacity ratio decay more seriously.

\section{CONCLUSION}

A series of PXDOT derivatives with different structures have been investigated. The presence of the bulky substitutions makes the PXDOT derivatives with more porous. The PProDOT shows a better electrochemical properties compared other polymers. This is due to its moderate porous structure which can enhance the ionic transfer and reduce the amount of ion pairs trapped within polymer in redox process. As a result, PProDOT can deliver a specific capacitance as high as $126 \mathrm{~F} \mathrm{~g}^{-1}$ and maintain $85 \%$ of its original capacitance after $1 \mathrm{k}$ cycles.

\section{Acknowledgements}

The author is grateful to the help from the Prof. Chu's group from RCAS, Academia Sinica, Taipei, Taiwan. 

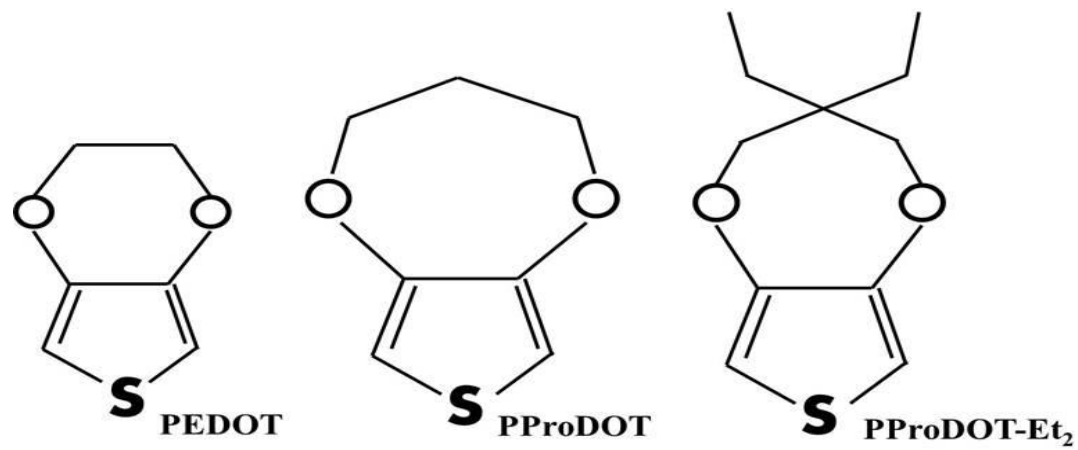

Scheme (1 ):-The Chemical structures of used polymers.
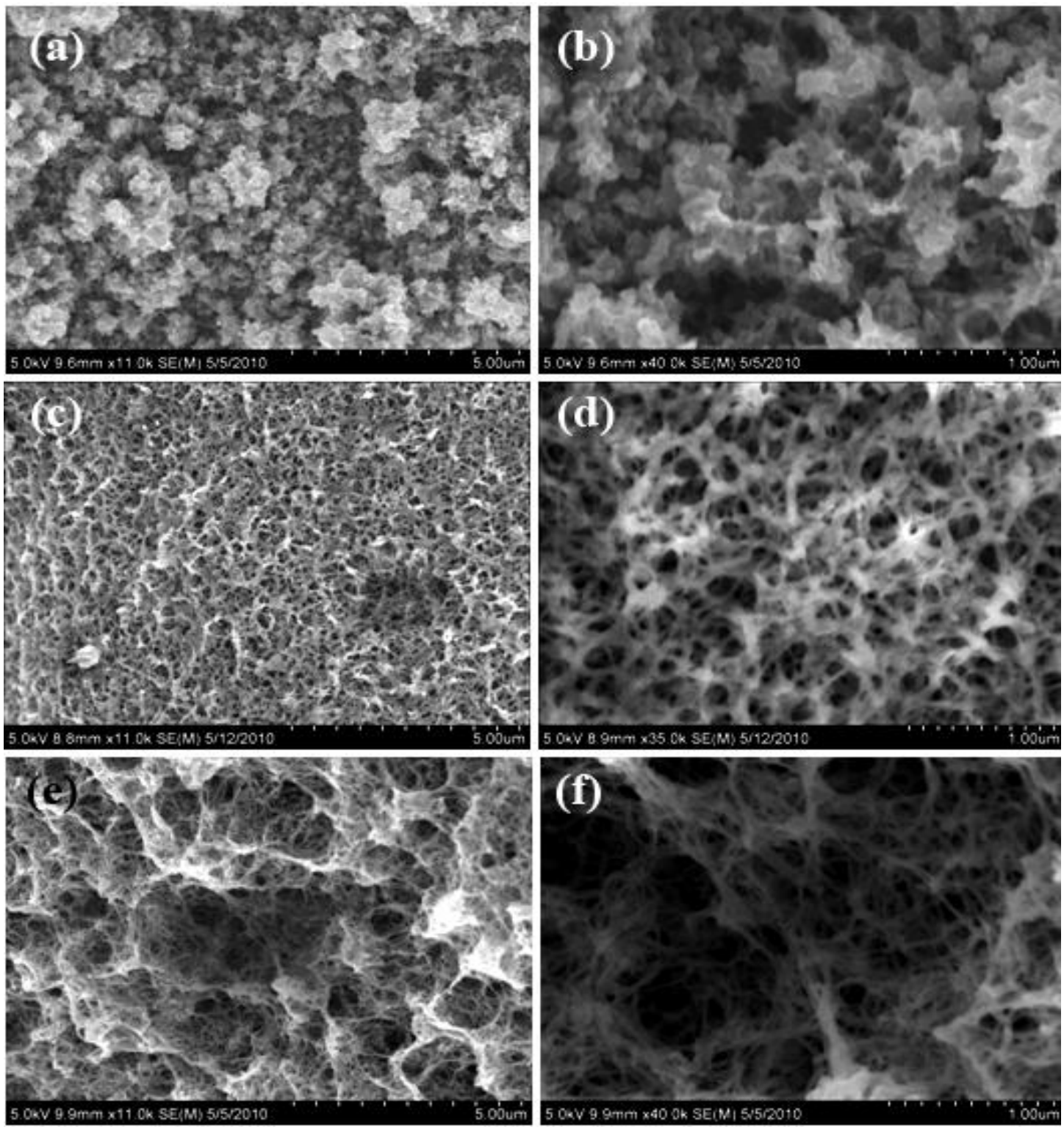

Fig. (1):- SEM surface morphology of PXDOTs. (a, b) PEDOT, (c, d) PProDOT, and (e, f) PProDOT-Et 2. 


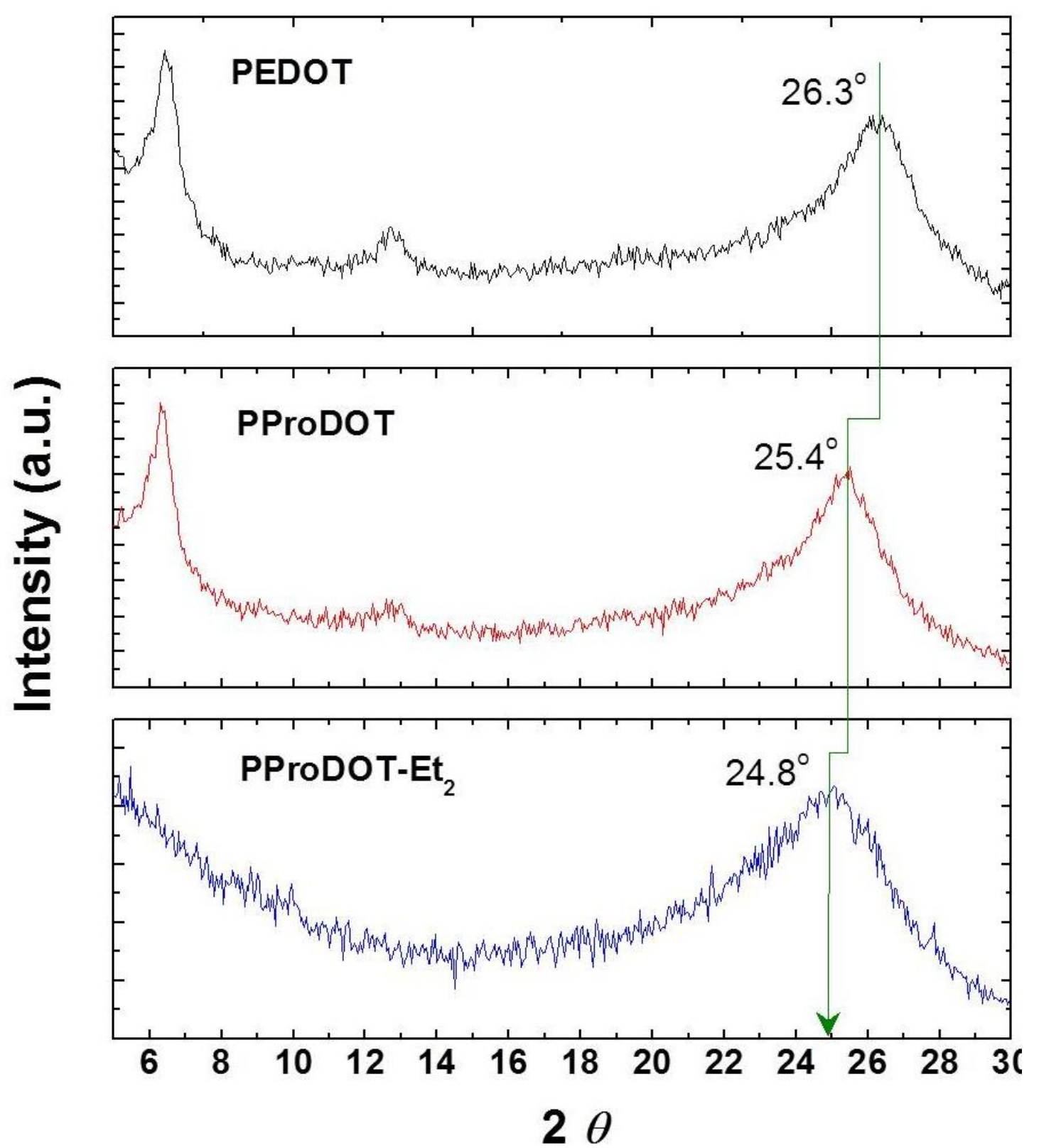

Fig.( 2 ):-XRD patterns of PXDOT powders prepared from electrochemical deposition. 


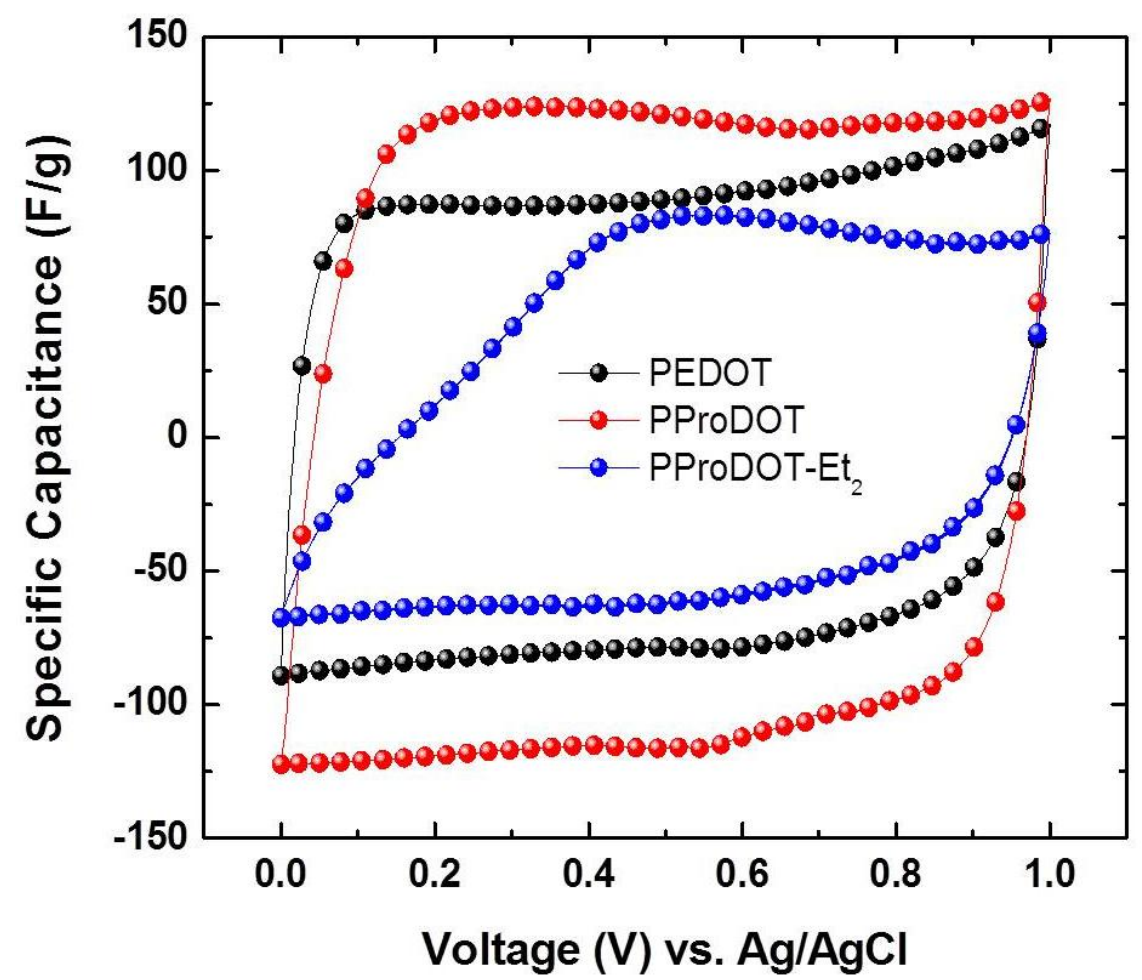

Fig. (3) :-Cyclic voltammograms of PXDOTs (vs. $\mathrm{Ag} / \mathrm{AgCl}$ ) in $1 \mathrm{M} \mathrm{LiClO}_{4}$ solution. The scan rate was $10 \mathrm{mV}$ $\mathrm{s}^{-1}$
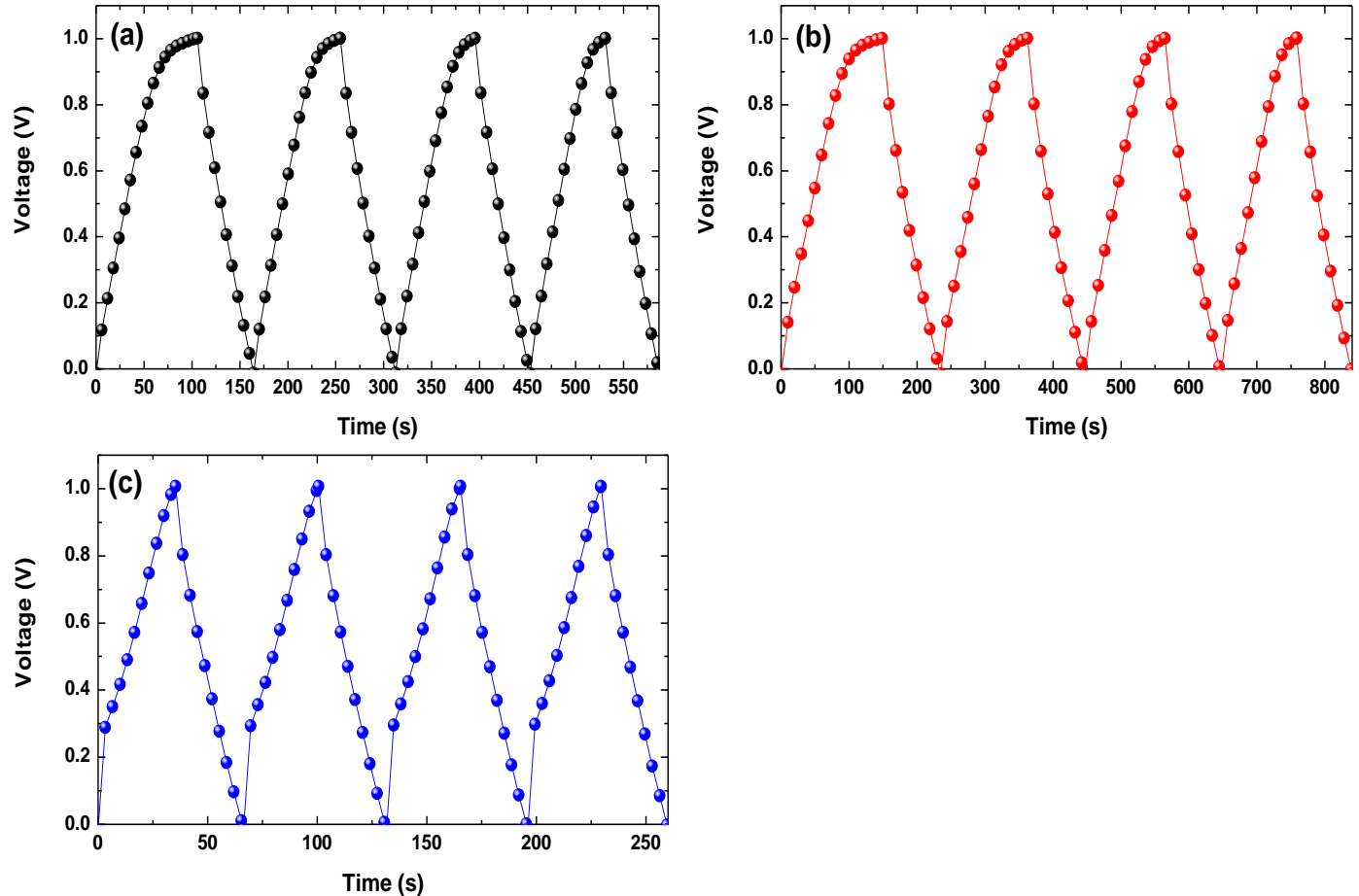

Fig.( 4):- Galvanostatic charge/discharge curves of PXDOTs. (a) PEDOT, (b) PProDOT, and (c) PProDOT-Et 2. 


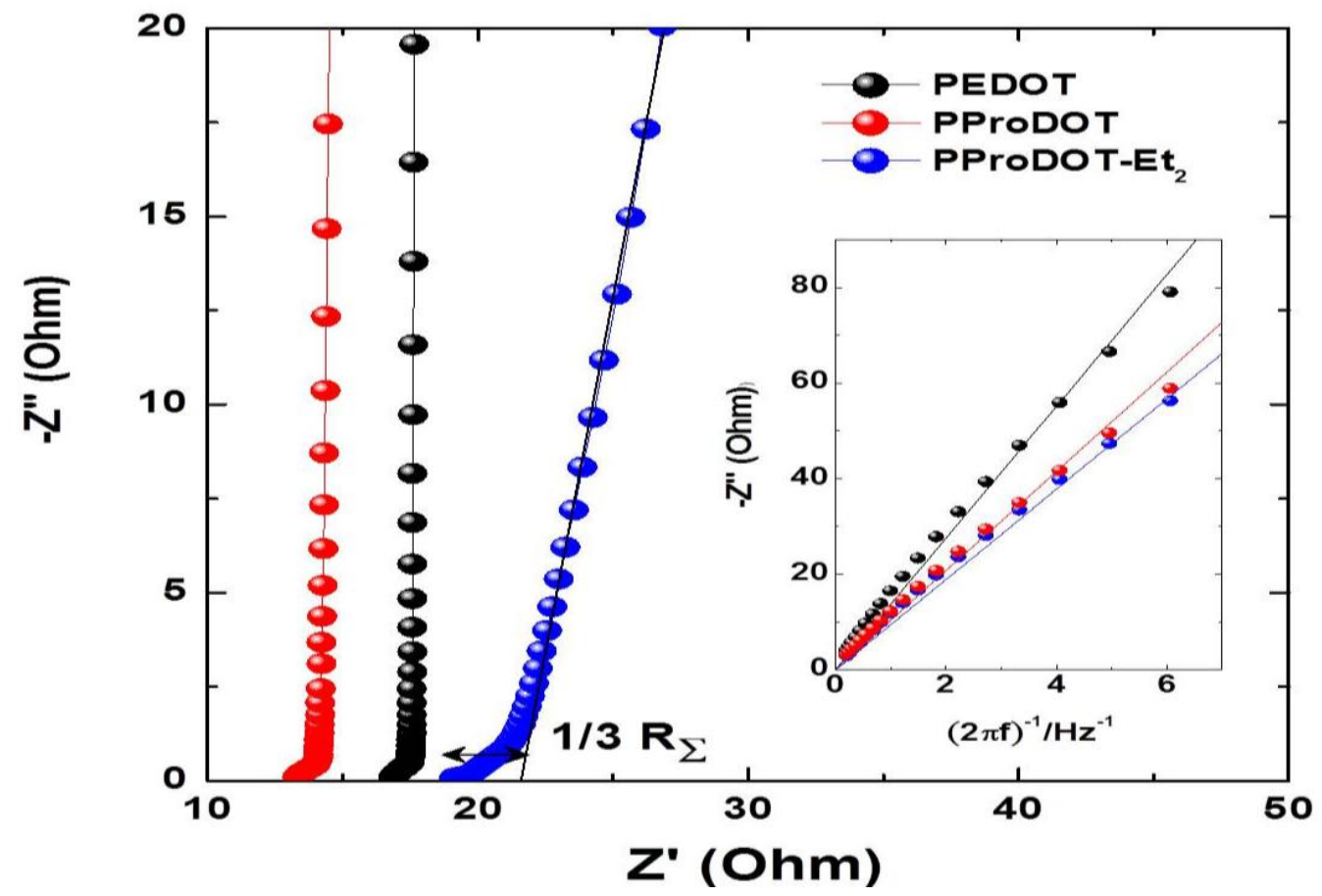

Fig. (5):- Impedance plot of PXDOTs in a various range of frequency from 0.01 to $10^{4} \mathrm{~Hz}$.
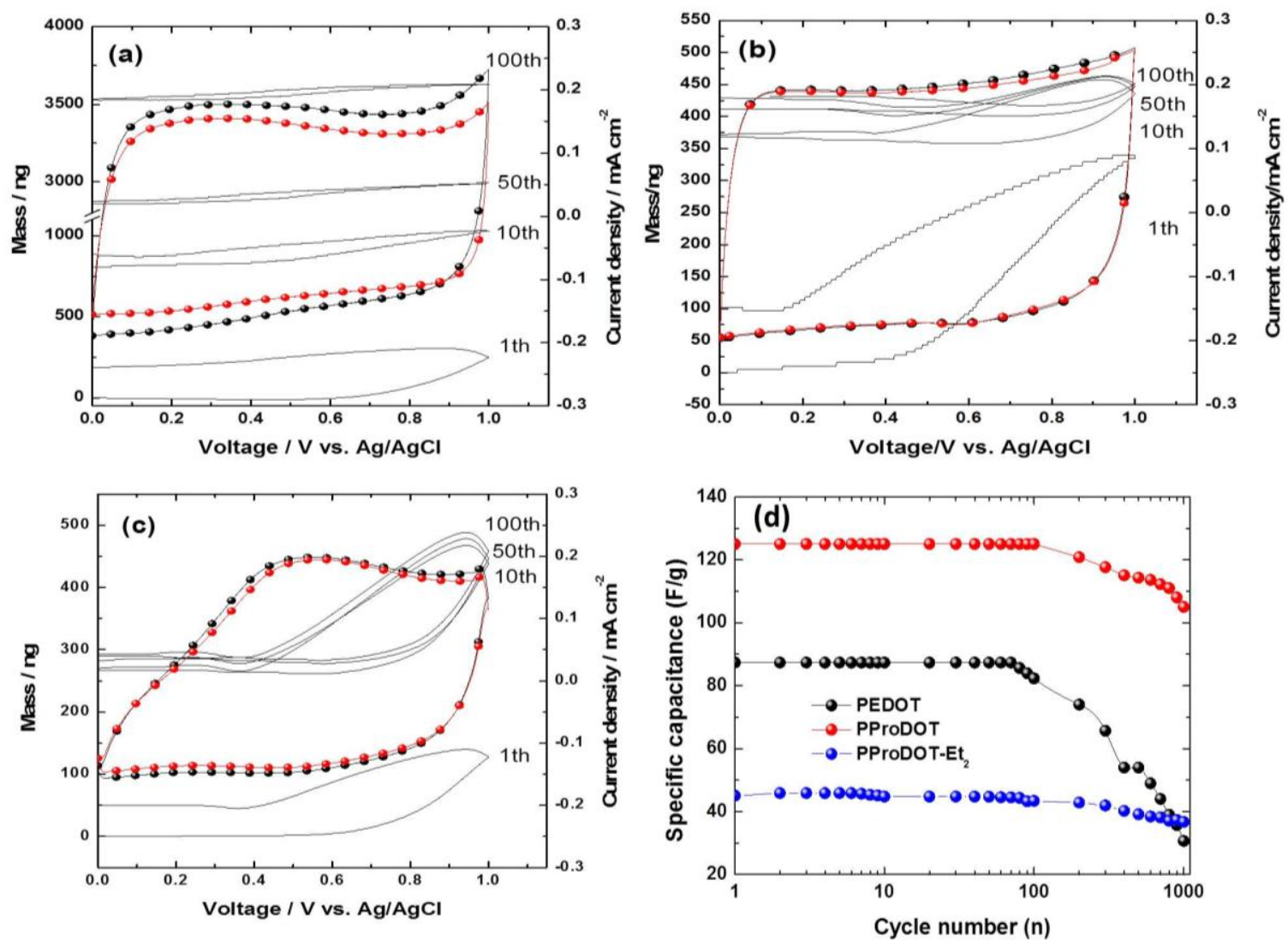

Fig. (6):- The EQCM and CV response of (a) PEDOT, (b) PProDOT, (c) PProDOT-Et ${ }_{2}$ in $1 \mathrm{M} \mathrm{LiClO}_{4}$ solution, and (d) Cycling test of PXDOTs at $1.5 \mathrm{~A} \mathrm{~g}^{-1}$ in $1 \mathrm{M} \mathrm{LiClO}_{4}$. 


\section{REFERENCES}

- M. Beidaghi, Y. Gogotsi, Energy \& Environmental Science, 7, 867 (2014).

-P. Simon, Y. Gogotsi, Nat Mater, 7 (2008) 845-854.

-Y. Xu, S. Jin, H. Xu, A. Nagai, D. Jiang, Chemical Society Reviews, 42 (2013) 8012-8031.

-V.T. Le, H. Kim, A. Ghosh, J. Kim, J. Chang, Q.A. Vu, D.T. Pham, J.-H. Lee, S.-W. Kim, Y.H. Lee, ACS Nano, 7 (2013) 5940-5947.

-X. Xiao, T. Li, Z. Peng, H. Jin, Q. Zhong, Q. Hu, B. Yao, Q. Luo, C. Zhang, L. Gong, J. Chen, Y. Gogotsi, J. Zhou, Nano Energy, 6 (2014) 1-9.

-B. Saravanakumar, K.K. Purushothaman, G. Muralidharan, ACS Applied Materials \& Interfaces, 4 (2012) 4484-4490.

-Y.G. Zhu, Y. Wang, Y. Shi, J.I. Wong, H.Y. Yang, Nano Energy, 3 (2014) 46-54.

-Y. Yang, S. Li, L. Zhang, J. Xu, W. Yang, Y. Jiang, ACS Applied Materials \& Interfaces, 5 (2013) 4350-4355.

-B.N. Reddy, M. Deepa, A.G. Joshi, Physical Chemistry Chemical Physics, 16 (2014) 2062-2071.

-Z. Su, C. Yang, C. Xu, H. Wu, Z. Zhang, T. Liu, C. Zhang, Q. Yang, B. Li, F. Kang, Journal of Materials Chemistry A, 1 (2013) 12432-12440.

-Y. Liu, B. Zhang, Y. Yang, Z. Chang, Z. Wen, Y. Wu, Journal of Materials Chemistry A, 1 (2013) 13582-13587.

-G.A. Snook, P. Kao, A.S. Best, Journal of Power Sources, 196 (2011) 1-12.
-Q. Wu, Y. Xu, Z. Yao, A. Liu, G. Shi, ACS Nano, 4 (2010) 1963-1970.

-R. Liu, S.I. Cho, S.B. Lee, Nanotechnology, 19 (2008) 215710.

-Y. Li, B. Wang, H. Chen, W. Feng, Journal of Power Sources, 195 (2010) 3025-3030.

-T.L. Kelly, K. Yano, M.O. Wolf, ACS Applied Materials \& Interfaces, 1 (2009) 2536-2543.

-T. Torimoto, T. Tsuda, K.-i. Okazaki, S. Kuwabata, Advanced Materials, 22 (2010) 1196-1221.

-X. Ren, P.G. Pickup, J. Electroanal. Chem. 372 (1994) 289.

-H. Ling, J. Lu, S. Phua, H. Liu, L. Liu, Y. Huang, D. Mandler, P.S. Lee, X. Lu, Journal of Materials Chemistry A, 2 (2014) 2708-2717.

-T.-A. Chen, X. Wu, R.D. Rieke, Journal of the American Chemical Society, 117 (1995) 233-244.

-R. Lin, P.L. Taberna, J. Chmiola, D. Guay, Y. Gogotsi, P. Simon, Journal of The Electrochemical Society, 156 (2009) A7.

-J.-H. Huang, Z.-Y. Ho, D. Kekuda, C.-W. Chu, K.-C. Ho, The Journal of Physical Chemistry C, 112 (2008) 19125-19130.

-M. Ates, Int. J. Electrochem. Sci.,4 (2009) 980 - 992.

-A. Robert Hillman, S.J. Daisley, S. Bruckenstein, Physical Chemistry Chemical Physics, 9 (2007) 2379-2388.

-W. Plieth, A. Bund, U. Rammelt, S. Neudeck, L. Duc, Electrochimica Acta, 51 (2006) 2366-2372. 